\title{
String perturbation theory around dynamically shifted vacuum
}

\author{
Roji Pius, ${ }^{a}$ Arnab Rudra ${ }^{b}$ and Ashoke Sen ${ }^{a}$ \\ ${ }^{a}$ Harish-Chandra Research Institute, \\ Chhatnag Road, Jhusi, Allahabad 211019, India \\ ${ }^{b}$ Department of Applied Mathematics and Theoretical Physics, \\ Wilberforce Road, Cambridge CB3 OWA, U.K. \\ E-mail: rojipius@mri.ernet.in, A.Rudra@damtp.cam.ac.uk, \\ sen@mri.ernet.in
}

Abstract: In some string theories, e.g. SO(32) heterotic string theory on Calabi-Yau manifolds, a massless field with a tree level potential can acquire a tachyonic mass at the one loop level, forcing us to quantize the theory around a new background that is not a solution to the classical equations of motion and hence is not described by a conformally invariant world-sheet theory. We describe a systematic procedure for carrying out string perturbation theory around such backgrounds.

Keywords: Superstrings and Heterotic Strings, Bosonic Strings

ArXiv EPRINT: 1404.6254 


\section{Contents}

1 Introduction 1

2 Systematic construction of the new vacuum 3

3 General amplitudes at the new vacuum $\quad 8$

4 Extension to superstring and heterotic string theories 12

5 Spurious infrared divergences $\quad 12$

$\begin{array}{ll}\text { A Effect of shifting a massless field } & 16\end{array}$

\section{Introduction}

In many $\mathcal{N}=1$ supersymmetric compactification of string theory down to $3+1$ dimensions, we have U(1) gauge fields with Fayet-Iliopoulos (FI) terms generated at one loop [1-4] (see $[5,6]$ for a recent perspective on this). By choosing suitable linear combination of these gauge fields we can ensure that only one gauge field has FI term. Typically there are also massless scalars $\phi_{i}$ charged under this U(1) gauge field. If $q_{i}$ is the charge carried by $\phi_{i}$ then the presence of the FI term generates a term in the potential of the form

$$
\frac{1}{g^{2}}\left(\sum_{i} q_{i} \phi_{i}^{*} \phi_{i}-C g^{2}\right)^{2}
$$

where $C$ is a numerical constant that determines the coefficient of the FI term and $g$ is the string coupling. $C$ could be positive or negative and $q_{i}$ 's for different fields could have different signs. As a result when we expand the potential in powers of $\phi_{i}$ around the perturbative vacuum $\phi_{i}=0$, some of these scalars can become tachyonic. ${ }^{1}$ It is clear from the form of the effective potential that the correct procedure to compute physical quantities is to shift the corresponding fields so that we have a new vacuum where $\sum_{i} q_{i}\left\langle\phi_{i}^{*}\right\rangle\left\langle\phi_{i}\right\rangle=$ $C g^{2}$, and quantize string theory around this new background. However since classically the $C g^{2}$ term is absent from this potential (1.1), this new vacuum is not a solution to the classical equations of motion. As a result on-shell methods [7-22], which require that we begin with a conformally invariant world-sheet theory, is not suitable for carrying out a systematic perturbation expansion around this new background.

\footnotetext{
${ }^{1}$ It was shown in [2] that for any compactification of $\mathrm{SO}(32)$ heterotic string theory preserving $(2,2)$ world-sheet supersymmetry, there is always at least one such tachyonic scalar, leading to the existence of a stable supersymmetric vacuum.
} 
Although the above example provides the motivation for our analysis, we shall address this in a more general context. At the same time we shall simplify our analysis by assuming that only one scalar field is involved instead of multiple scalar fields. So we consider a general situation in string theory where at tree level we have a massless real scalar with a non-zero four point coupling represented by a potential

$$
A \phi^{4}+\cdots
$$

where $\cdots$ denote higher order terms. We suppose further that at one loop the scalar receives a negative contribution $-2 C g^{2}$ to its mass ${ }^{2}$. Here $A$ and $C$ are $g$-independent constants. Then the total potential will be

$$
A \phi^{4}-C g^{2} \phi^{2}+\cdots
$$

This has a minimum at

$$
\phi^{2}=\frac{1}{2} \frac{C}{A} g^{2}+\cdots .
$$

Our goal will be to understand how to systematically develop string perturbation theory around this new background and also to correct the expectation value of $\phi$ due to higher order corrections. If we had an underlyng string field theory that is fully consistent at the quantum level, e.g. the one described in [23], then that would provide a natural framework for addressing this issue. Our method does not require the existence of an underlying string field theory, although the requirement of gluing compatibility of the local coordinate system that we shall use is borrowed from string field theory.

The method we shall describe can be used to address other similar problems in string theory where loop correction induces small shift in the vev of a massless field. For example suppose we have a massless field $\chi$ with a tree level cubic potential and suppose further that one loop correction generates a tadpole for this field. Then from the effective field theory approach it is clear that there is a nearby perturbative vacuum where the field $\chi$ is non-tachyonic. Usual string perturbation theory does not tell us how to deal with this situation, but the method we describe below can be used in this case as well.

There are of course also problems involving tadpoles of massless fields without tree level potential, e.g. of the kind discussed in [24, 25] and many follow up papers. As of now our method does not offer any new insight into such problems.

Earlier attempts to analyze string theory in a shifted background can be found in [30]. However unlike in [30], here by focussing on a concrete class of problems where the shift is perturbative in the string coupling, and employing a stringy infrared regulator that renders diagrams with arbitrary number of tadpoles finite at the intermediate steps, we shall give a completely systematic procedure for computing amplitudes in the shifted vacuum to any given order in the perturbation theory.

The rest of the paper is organised as follows. In section 2 we describe the procedure for constructing amplitudes in the presence of a small shift in the vacuum expectation value of a massless scalar following the procedure of [26-29]. We also discuss systematic procedure for determining the shift by requiring absence of tadpoles. In section 3 we show that the amount of shift in the scalar, needed to cancel the tadpole, depends on the choice of 
local coordinate system that we use to construct the amplitudes. However general physical amplitudes in the presence of the shift are independent of the choice of local coordinate system as long as we use a gluing compatible system of local coordinates for defining the amplitude. This is our main result. For simplicity we restrict our analysis in section 2 and section 3 to bosonic string theory, but in section 4 we discuss generalization of our analysis to include NS sector states in heterotic and superstring theories. In section 5 we describe the procedure for regulating the spurious infrared divergences in loops, arising from the fact that the shift in the vacuum renders some of the originally massless states massive.

\section{Systematic construction of the new vacuum}

We shall carry out our analysis under several simplifying assumptions. These are made mainly to keep the analysis simple, but we believe that none of these (except 4) is necessary.

1. We shall assume that there is a symmetry under which $\phi \rightarrow-\phi$ so that amplitudes with odd number of external $\phi$ fields vanish.

2. We shall assume that $\phi$ does not mix with any other physical or unphysical states of mass level zero even when quantum corrections are included.

3. Shifting the $\phi$ field can sometimes induce tadpoles in other massless fields. If there is a tree level potential for this field then we can cancel the tadpole by giving a vacuum expectation value (vev) to that field and determine the required vev by following the same procedure that we used to determine the shift in $\phi$. We shall assume that such a situation does not arise and that $\phi$ is the only field that needs to be shifted. However extension of our analysis to this more general case should be straightforward.

4. If on the other hand shifting $\phi$ leads to the tadpole of a massless field which has vanishing tree level potential then it is not in general possible to find a nearby vacuum where all tadpoles vanish. In this case the vacuum is perturbatively unstable. We shall assume that this is not the case here.

5. When the theory has other massless fields besides $\phi$ but their tadpoles vanish, then the situation can be dealt with in the manner discussed in section 7.2 of [7] and will not be discussed here any further. ${ }^{2}$

6. In this section and in section 3 we shall restrict our analysis to the bosonic string theory. However the result can be generalized to include the case where $\phi$ is NeveuSchwarz (NS) sector field in the heterotic string theory or NS-NS sector field in type IIA or IIB string theory. This is discussed briefly in section 4 .

As discussed in detail in $[34,35]$, for computing renormalized masses and S-matrix of general string states we need to work with off-shell string theory. This requires choosing

\footnotetext{
${ }^{2}$ In the special case of the D-term potential in supersymmetric theories discussed in section 1 the dilaton tadpole does not vanish in the perturbative vacuum [1, 7, 31-33], but is expected to vanish in the shifted vacuum since the latter has zero energy density.
} 
a set of gluing compatible local coordinate system on the (super-)Riemann surfaces. The result for off-shell amplitude depends on the choice of local coordinates, but the renormalized masses and S-matrix elements computed from it are independent of this choice. Our analysis will be carried out in this context.

The off-shell amplitudes do not directly compute the off-shell Green's functions. Instead they compute truncated off-shell Green's functions. If we denote by $G^{(n)}\left(k_{1}, b_{1} ; \cdots k_{n}, b_{n}\right)$ the $n$-point off-shell Green's function of fields carrying quantum numbers $\left\{b_{i}\right\}$ and momenta $\left\{k_{i}\right\}$, then the truncated off-shell Green's functions are defined as

$$
\Gamma^{(n)}\left(k_{1}, b_{1} ; \cdots k_{n}, b_{n}\right)=G^{(n)}\left(k_{1}, b_{1} ; \cdots k_{n}, b_{n}\right) \prod_{i=1}^{n}\left(k_{i}^{2}+m_{b_{i}}^{2}\right),
$$

where $m_{b}$ is the tree level mass of the state carrying quantum number $b$. We shall use only connected component of the Green's function in the definition of $\Gamma^{(n)}$ and remove from it the overall momentum conserving delta function, at the same time choosing the external momenta such that their sum vanishes. The usual on-shell amplitudes of string theory compute $\Gamma^{(n)}$ at $k_{i}^{2}=-m_{b_{i}}^{2}$. This differs from the S-matrix elements by multiplicative wave-function renormalization factors for each external state and also due to the fact that the S-matrix elements require replacing $m_{b_{i}}^{2}$ 's by physical mass ${ }^{2}$ 's in this formula. However from the knowledge of off-shell amplitude $\Gamma^{(n)}$ defined in $(2.1)$ we can extract the physical S-matrix elements following the procedure described in [34, 35].

Our goal is to study what happens when we switch on a vev of $\phi$. For this we shall first consider a slightly different situation. Suppose that $\phi$ is an exactly marginal deformation in string theory and furthermore that it remains marginal even under string loop corrections. In this case there is no potential for $\phi$ and we can give any vacuum expectation value $\lambda$ to $\phi$. The effect of this is to deform the world-sheet action by a term $\lambda \int d^{2} z V_{\phi}(z, \bar{z})$ where $V_{\phi}$ is the vertex operator of the zero momentum $\phi$ state. In the string amplitude, obtained by integrating the correlation functions of the underlying conformal field theory (CFT) on moduli spaces of punctured Riemann surfaces, this introduces a term

$$
\exp \left[\lambda \int d^{2} z V_{\phi}(z, \bar{z})\right]=\sum_{m=0}^{\infty} \frac{\lambda^{m}}{m !}\left(\int d^{2} z V_{\phi}(z, \bar{z})\right)^{m} .
$$

The effect of the $\left(\int d^{2} z V_{\phi}(z, \bar{z})\right)^{m}$ term is to convert $\Gamma^{(n)}$ to $\Gamma^{(n+m)}$ with $m$ insertions of zero momentum $\phi$ state. This if we denote by $\Gamma_{\lambda}^{(n)}$ the deformed off-shell amplitudes then we have the relation

$$
\Gamma_{\lambda}^{(n)}\left(k_{1}, b_{1} ; \cdots k_{n}, b_{n}\right)=\sum_{m=0}^{\infty} \frac{\lambda^{m}}{m !} \Gamma^{(n+m)}\left(k_{1}, b_{1} ; \cdots k_{n}, b_{n} ; 0, \phi ; \cdots 0, \phi\right),
$$

where we have denoted the quantum number $b$ labelling the field $\phi$ by $\phi$ itself. There are altogether $m$ insertions of $0, \phi$ in the argument of $\Gamma^{(n+m)}$ on the right hand side. In any expression of this kind that we shall be using later, the number of insertions of $(0, \phi)$ can be figured out by subtracting from the superscript of $\Gamma$ the number of explicit $\left(k_{i}, b_{i}\right)$ factors in the argument of $\Gamma$. The insertion of $(0, \phi)$ factors in $(2.3)$ has to be interpreted 
as the result of taking the zero momentum limit of a general amplitude where the external $\phi$ states carry non-zero and different momenta. As we shall see, individual contributions to the right hand side have tadpole divergence in the zero momentum limit. We shall discuss ways to regulate this later. A direct proof of (2.3) in a quantum field theory has been given in appendix $\mathrm{A}$.

Now in our case the field $\phi$ has a potential even at the tree level and hence does not represent an exactly marginal deformation. If we nevertheless go ahead and try to define a deformed theory using (2.3), we encounter the following problem. We have

$$
\Gamma_{\lambda}^{(1)}(0, \phi)=\sum_{\substack{m=0 \\ m \text { odd }}}^{\infty} \frac{\lambda^{m}}{m !} \Gamma^{(1+m)}(0, \phi ; 0, \phi ; \cdots 0, \phi),
$$

where we have used the postulated $\phi \rightarrow-\phi$ symmetry to restrict the sum over $m$ to odd values only. It will be useful to express the right hand side as sum over contributions from different genera. Thus we write ${ }^{3}$

$$
\Gamma_{\lambda}^{(1)}(0, \phi)=\sum_{s=0}^{\infty} g^{2 s} \sum_{\substack{m=0 \\ m \text { odd } m+2 s \geq 3}}^{\infty} \frac{\lambda^{m}}{m !} \Gamma^{(1+m ; s)}(0, \phi ; 0, \phi ; \cdots 0, \phi)
$$

where $\Gamma^{n ; s}$ denotes genus $s$ contribution to the $n$-point function in the unperturbed theory. The $s=0, m=3$ term on the right hand side is non-zero since it is proportional to the four point function of zero momentum $\phi$ states and is proportional to $A$ according to (1.2). Thus in the deformed theory there is a zero momentum $\phi$ tadpole proportional to $\lambda^{3}$. This is clearly not an acceptable vacuum at the tree level since it will give divergent results for higher point amplitudes.

But now consider the effect of one loop correction given by the $m=1, s=1$ term on the right hand side of (2.5). This is non-zero and represents the second term in (1.3). We now see that by a suitable choice of $\lambda$, given by the solution to

$$
\frac{1}{6} \lambda^{3} \Gamma^{(4 ; 0)}(0, \phi ; 0, \phi ; 0, \phi ; 0, \phi)+\lambda g^{2} \Gamma^{(2 ; 1)}(0, \phi ; 0, \phi)=0
$$

we can cancel the net contribution to the $\phi$ tadpole to order $g^{3}$. This vanishes for three distinct values of $\lambda$, one of which is given by $\lambda=0$ and the other two are related by $\phi \rightarrow-\phi$ symmetry. We shall be considering the situation where in the $\lambda=0$ vacuum the field $\phi$ is tachyonic and hence this solution needs to be avoided. This fixes $\lambda$ to a specific value of order $g$ up to the $\phi \rightarrow-\phi$ symmetry.

\footnotetext{
${ }^{3}$ We have dropped an overall $1 / g^{2}$ factor from the definition of $\Gamma^{(n)}$ so that we can drop a $g^{2}$ factor from the definition of the propagator later in (2.9). If we use the standard convention where $1 / g^{2}$ appears as an overall multiplicative factor in the tree level action, then the propagator $\Delta$ will have an extra factor of $g^{2}$ and the $\Gamma^{(n)}$ 's will have extra factor of $1 / g^{2}$. If we denote these by $\Gamma_{\text {standard }}=g^{-2} \Gamma$ and $\Delta_{\text {standard }}=g^{2} \Delta$, then it is straightforward to check that all our subsequent equations hold with $\Gamma$ replaced by $\Gamma_{\text {standard }}$ and $\Delta$ replaced by $\Delta_{\text {standard }}$ without any extra factor of $g^{2}$.
} 
With this choice of $\lambda$ we make $\Gamma_{\lambda}^{(1)}(0, \phi)$ vanish to order $g^{3}$. To extend the analysis to higher order in $g$ we express the condition of vanishing of (2.5) as

$$
\begin{aligned}
\frac{1}{6} \lambda^{2} & \Gamma^{(4 ; 0)}(0, \phi ; 0, \phi ; 0, \phi ; 0, \phi)+g^{2} \Gamma^{(2 ; 1)}(0, \phi ; 0, \phi) \\
& =-\sum_{\substack{m, s=0 \\
m \text { odd } m+2 s \geq 5}}^{\infty} \frac{1}{m !} \lambda^{m-1} g^{2 s} \Gamma^{(1+m ; s)}(0, \phi ; 0, \phi ; \cdots 0, \phi)
\end{aligned}
$$

and then solve this equation iteratively using the leading order solution (2.6) as the starting point. Note that in arriving at (2.7) we have divided (2.5) by $\lambda$, thereby removing the trivial solution $\lambda=0$. To each order in iteration we substitute on the right hand side of (2.7) the solution for $\lambda$ to the previous order and then solve (2.7). Due to the $\phi \rightarrow-\phi$ symmetry and the fact that the genus expansion is in powers of $g^{2}$, the solution for $\lambda$ takes the form

$$
\lambda^{2}=\sum_{n=0}^{\infty} A_{n} g^{2 n+2},
$$

for constants $A_{n}$. Furthermore note that by adjusting $\lambda^{2}$ to order $g^{2 n+2}$, we can satisfy (2.7) to terms of order $g^{2 n+2}$, i.e. make the right hand side of (2.5) vanish to order $g^{2 n+3} .4$

There are however some additional subtleties in this analysis, since the individual terms on the right hand side of (2.7) can diverge due to $\phi$ tadpole. These divergences arise from regions of moduli integral where a Riemann surface degenerates into two distinct Riemann surfaces connected by a long handle. As mentioned at the end of section 1, we shall proceed with the assumption that the only relevant divergences are associated with tadpoles of $\phi$. To deal with these divergences we need to first regularize these divergences, solve for $\lambda$, and at the end remove the regulator. For this we shall work with a choice of gluing compatible local coordinates according to the procedure described in section 3.2 of [35] and express a general amplitude contributing to the right hand side of (2.4) as a sum of products of 'one particle irreducible' (1PI) amplitudes joined by the propagator

$$
\Delta=\frac{1}{4 \pi} \int_{0}^{\infty} d s \int_{0}^{2 \pi} d \theta e^{-(s-i \theta) L_{0}-(s+i \theta) \bar{L}_{0}} .
$$

The divergence in individual contributions come from the $s \rightarrow \infty$ limit on the right hand side of (2.9). We shall regulate the divergence by replacing $\Delta$ by

$$
\frac{1}{4 \pi} \int_{0}^{\Lambda} d s \int_{0}^{2 \pi} d \theta e^{-(s-i \theta) L_{0}-(s+i \theta) \bar{L}_{0}},
$$

where $\Lambda$ is a fixed large number. The relevant divergence in $\Delta$ comes from the choice where the propagating state is a zero momentum $\phi$. Since $L_{0}$ and $\bar{L}_{0}$ vanish for zero momentum

\footnotetext{
${ }^{4}$ Due to the $\phi \rightarrow-\phi$ symmetry and the fact that the genus expansion is in powers of $g^{2}$, the contributions to $\Gamma_{\lambda}^{(1)}(0, \phi)$ involve only odd powers of $g$ after using $(2.8)$. Thus making $\Gamma_{\lambda}^{(1)}(0, \phi)$ vanish to order $g^{2 n+3}$ also makes it vanish to order $g^{2 n+4}$.
} 


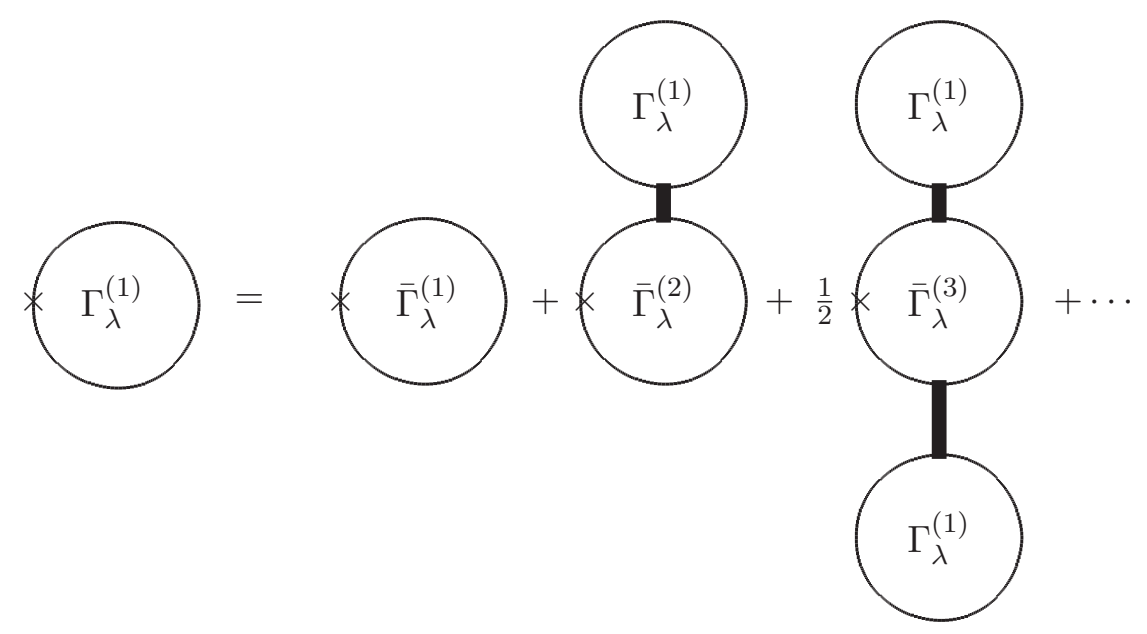

Figure 1. Pictorial representation of (2.12). The $\times$ denotes the vertex operator associated with the external $\phi$ state. The $\phi$ vertex operators carrying factors of $\lambda$ are not displayed explicitly. The thick line denotes the $\phi$ propagator $\Delta_{\phi}$.

$\phi$, the contribution to $\Delta$ from this term goes as $P_{\phi} \Lambda / 2$, where $P_{\phi}$ denotes projection to the CFT state corresponding to zero momentum $\phi$. It will be convenient to define, ${ }^{5}$

$$
\begin{aligned}
& \Delta_{\phi}=\frac{1}{2} \Lambda P_{\phi}, \quad \bar{\Delta}=\Delta-\Delta_{\phi} \quad \text { for momentum } \quad k=0, \\
& \bar{\Delta}=\Delta \quad \text { for } k \neq 0 \text {. }
\end{aligned}
$$

We furthermore denote by $\bar{\Gamma}$ the contribution to an amplitude obtained by taking sum of products of 1PI contributions joined by the modified propagator $\bar{\Delta}$. Thus the difference between the full amplitude $\Gamma$ and the modified amplitude $\bar{\Gamma}$ is controlled by $\Delta_{\phi}$. We expect the individual contributions to $\bar{\Gamma}$ to be free from divergence associated with zero momentum propagator in the $\Lambda \rightarrow \infty$ limit, since the contribution to $\bar{\Delta}$ from a massive state of mass $m$ is given by $\left(1-e^{-\Lambda m^{2} / 2}\right) / m^{2}$, while the contribution from the other massless states are expected to vanish due to the assumed vanishing of the corresponding tadpoles. From this argument it is also clear that the $\Lambda$ dependence of $\bar{\Gamma}$ will come through exponentially suppressed terms for large but finite $\Lambda$. The full amplitude is obtained as sum of products of $\bar{\Gamma}$ 's joined by $\Delta_{\phi}$ 's.

We now define $\bar{\Gamma}_{\lambda}^{(n)}$ as in (2.3) with all the $\Gamma$ 's replaced by $\bar{\Gamma}$ 's on the right hand side. This allows us to express $\Gamma_{\lambda}^{(1)}(0, \phi)$ as (see figure 1)

$$
\Gamma_{\lambda}^{(1)}(0, \phi)=\sum_{k=0}^{\infty} \frac{1}{k !} \bar{\Gamma}_{\lambda}^{(1+k)}(0, \phi ; 0, \phi ; \cdots ; 0, \phi)\left(\Delta_{\phi} \Gamma_{\lambda}^{(1)}(0, \phi)\right)^{k} .
$$

By repeated use of (2.12) we can express $\Gamma_{\lambda}^{(1)}(0, \phi)$ as sum of products of $\bar{\Gamma}_{\lambda}$ factors and $\Delta_{\phi}$ 's, but we shall not write down the explicit formula.

\footnotetext{
${ }^{5}$ For computation of $\Gamma_{\lambda}^{(1)}$ using $(2.5)$ all propagators carry zero momentum and hence the second line of (2.11) is irrelevent, but this definition will be useful for analyzing $\Gamma_{\lambda}^{(n)}$ in the next section.
} 
Now suppose that $\lambda$ to order $g^{2 n+1}$, obtained by solving (2.7) to order $g^{2 n+2}$, or equivalently by demanding the vanishing of $\Gamma_{\lambda}^{(1)}(0, \phi)$ to order $g^{2 n+3}$, has finite limit as $\Lambda \rightarrow \infty$. Our goal will be to prove that the result also holds with $n$ replaced by $n+1$. For determining $\lambda$ to next order, we need to compute $\Gamma_{\lambda}^{(1)}(0, \phi)$ to order $g^{2 n+5}$ and then require this to vanish. Using the result that $\bar{\Gamma}_{\lambda}^{(2)}(0, \phi ; 0, \phi)$ has its expansion beginning at order $g^{2}$, one can show that in order to compute the right hand side of (2.12) to order $g^{2 n+5}$ we need to know the $\Gamma_{\lambda}^{(1)}(0, \phi)$ appearing on the right hand side of $(2.12)$ at most to order $g^{2 n+3}$. By assumption this contribution vanishes. Thus only the $k=0$ term contributes on the right hand side of $(2.12)$, showing that to order $g^{2 n+5}, \Gamma_{\lambda}^{(1)}(0, \phi)=\bar{\Gamma}_{\lambda}^{(1)}(0, \phi)$. This means that in order to determine the order $g^{2 n+3}$ correction to $\lambda$ we can replace $\Gamma$ by $\bar{\Gamma}$ on the right hand side of (2.7). ${ }^{6}$ Since $\bar{\Gamma}$ 's by construction are finite as $\Lambda \rightarrow \infty$ we see that the order $g^{2 n+3}$ correction to $\lambda$ is also finite as $\Lambda \rightarrow \infty$. This proves the desired result.

We shall see in section 3 that even though $\lambda$ determined using this procedure is finite in the $\Lambda \rightarrow \infty$ limit, it is ambiguous i.e. it depends on the choice of local coordinate system used for the computation. Nevertheless all physical amplitudes will turn out to be free from this ambiguity.

\section{General amplitudes at the new vacuum}

Once $\lambda$ is determined, we can use (2.3) to compute the general $n$-point amplitude in the deformed theory. However we need to ensure that this has finite $\Lambda \rightarrow \infty$ limit and that it is unambiguous, e.g. independent of the choice of local coordinates used to define the 1PI amplitudes. Our discussion will follow closely that of section 7.6 of [7]. However in [7] the massless tadpoles were assumed to cancel at every genus while here we consider the case where the cancelation is between the contributions from different genera. Furthermore in [7] the massless fields were assumed to have flat potential while here the relevant field $\phi$ has a potential even at the tree level.

First we examine the issue of finiteness in the $\Lambda \rightarrow \infty$ limit. We shall assume that all the external states (labelled by $1, \cdots n$ in $(2.3)$ ) carry generic non-zero momentum. Thus possible source of zero momentum propagators on the right hand side of (2.3) are propagators which connect two Riemann surfaces, one of which carry all the external states $1, \cdots n$ and possibly some of the zero momentum $\phi$ vertex operators and the other one carries only zero momentum $\phi$ vertex operators. For studying the divergences associated with these zero momentum propagators, we shall define $\bar{\Delta}$ as in (2.11) and introduce the amplitudes $\bar{\Gamma}$ by following the procedure described below (2.11). It is easy to see that we now have the following generalization of (2.12) (see figure 2)

$$
\Gamma_{\lambda}^{(n)}\left(k_{1}, b_{1} ; \cdots k_{n}, b_{n}\right)=\sum_{k=0}^{\infty} \frac{1}{k !} \bar{\Gamma}_{\lambda}^{(n+k)}\left(k_{1}, b_{1} ; \cdots k_{n}, b_{n} ; 0, \phi ; \cdots 0, \phi\right)\left(\Delta_{\phi} \Gamma_{\lambda}^{(1)}(0, \phi)\right)^{k} .
$$

Now $\lambda$ has been chosen so that $\Gamma_{\lambda}^{(1)}(0, \phi)$ vanishes. This shows that only the $k=0$ term contributes to the right hand side of (3.1). Since $\bar{\Gamma}_{\lambda}^{(n)}\left(k_{1}, b_{1} ; \cdots k_{n}, b_{n}\right)$ is finite as $\Lambda \rightarrow \infty$, this establishes that $\Gamma_{\lambda}^{(n)}\left(k_{1}, b_{1} ; \cdots k_{n}, b_{n}\right)$ also has a finite limit as $\Lambda \rightarrow \infty$.

\footnotetext{
${ }^{6} \Gamma$ 's appearing on the left hand side of $(2.7)$ are in any case equal to the corresponding $\bar{\Gamma}$ 's.
} 


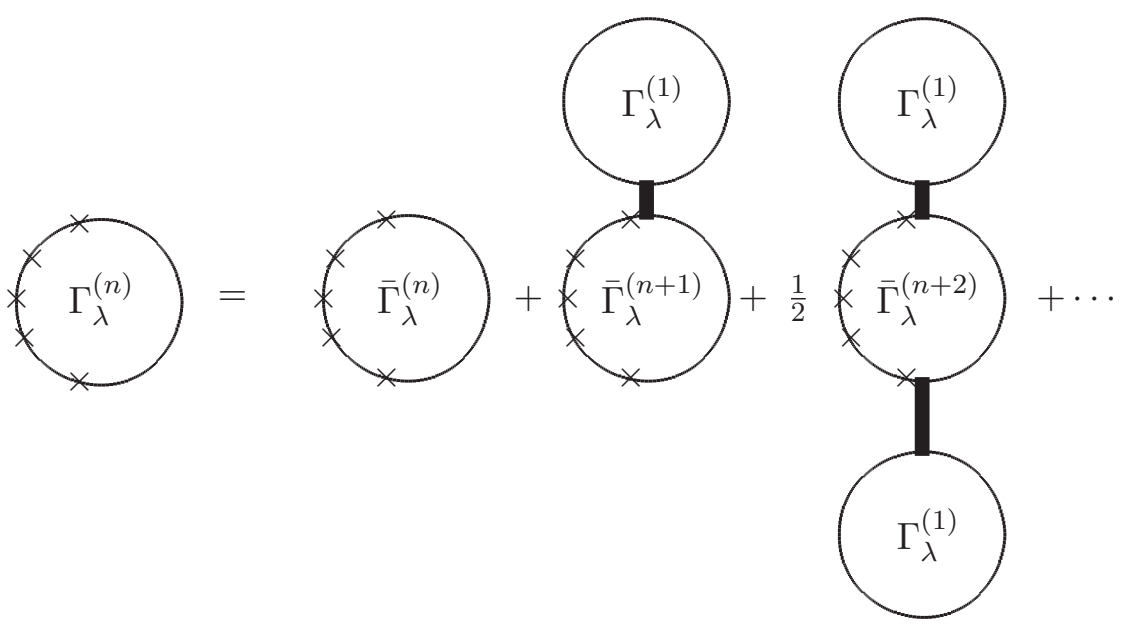

Figure 2. Pictorial representation of (3.1). The $\times$ denotes the vertex operator associated with the external states carrying quantum numbers $\left(k_{1}, b_{1} ; \cdots k_{n}, b_{n}\right)$. The $\phi$ vertex operators carrying factors of $\lambda$ are not displayed explicitly. The thick line denotes the propagator $\Delta_{\phi}$.

We now have to show that $\Gamma_{\lambda}^{(n)}\left(k_{1}, b_{1} ; \cdots k_{n}, b_{n}\right)$ is independent of the choice of local coordinate system, except for the expected dependence associated with the off-shell external states carrying momenta $k_{1}, \cdots k_{n}$. These latter dependences can be analyzed and treated in the same way as in $[34,35]$, and we shall not discuss them any further. To focus on the real issue we can for example concentrate on the case where these external states are massless states that do not suffer from mass renormalization so that the corresponding vertex operators do not introduce any dependence on the choice of local coordinates. ${ }^{7}$ The problematic dependence on local coordinates arises from the following source [7] (section 7.6). Let us consider two Riemann surfaces $A$ and $B$, glued at their punctures $P_{1}$ and $P_{2}$ by plumbing fixture procedure:

$$
w_{1} w_{2}=e^{-s+i \theta}
$$

where $w_{1}$ and $w_{2}$ are the local coordinates around the punctures $P_{1}$ and $P_{2}$ and $(s, \theta)$ are the same variables which appear in the definition (2.9) of the propagator. Having the cut-off $s \leq \Lambda$ then corresponds to requiring

$$
\left|w_{1} w_{2}\right| \geq e^{-\Lambda}
$$

Now suppose we change the local coordinates to $w_{1}^{\prime}, w_{2}^{\prime}$ related to $w_{1}$ and $w_{2}$ via the relations

$$
w_{1}=f\left(w_{1}^{\prime}\right), \quad w_{2}=g\left(w_{2}^{\prime}\right),
$$

where $f$ and $g$ are some specific functions satisfying $f(0)=0, g(0)=0$. Since for small $w_{1}$ and $w_{2}$ we have

$$
w_{1}=f^{\prime}(0) w_{1}^{\prime}, \quad w_{2}=g^{\prime}(0) w_{2}^{\prime},
$$

\footnotetext{
${ }^{7}$ The wave-function renormalization factors do depend on the choice of local coordinates, but this can be treated as in [7].
} 
we can express (3.3) as

$$
\left|w_{1}^{\prime} w_{2}^{\prime}\right| \geq e^{-\Lambda^{\prime}}, \quad \Lambda^{\prime}=\Lambda+2 \xi_{A}+2 \xi_{B}, \quad \xi_{A} \equiv \frac{1}{2} \ln \left|f^{\prime}(0)\right|, \quad \xi_{B} \equiv \frac{1}{2} \ln \left|g^{\prime}(0)\right| .
$$

Here $A$ and $B$ refer to the two Riemann surfaces that are connected by the propagator whose change we are considering. $A$ and $B$ are abstract symbols which characterize information on the external legs, genus, as well as the point in the moduli space we are in, since $f^{\prime}(0)$ and $g^{\prime}(0)$ could depend on all these informations. Note however that $\xi_{A}$ does not depend on the Riemann surface $B$ and $\xi_{B}$ does not depend on the Riemann surface $A$ provided we choose a gluing compatible local coordinate system.

(3.6) shows that changing the local coordinates correspond to effectively changing the cut-off. This in turn changes the regulated propagator (2.10) by

$$
\delta_{\Lambda} \Delta=\left(\xi_{A}+\xi_{B}\right) P_{\phi}
$$

We have ignored the change in the propagator due to massive states since they are exponentially suppressed in the $\Lambda \rightarrow \infty$ limit, and also from other massless states with vanishing tadpole since their effect can be taken care of by following the procedure described in [7] (see also [36]). This justifies the appearance of the projection operator $P_{\phi}$ to zero momentum $\phi$ states.

Now consider the right hand side of (2.7). Individual terms in this expression are divergent in the $\Lambda \rightarrow \infty$ limit, but when we use the value of $\lambda$ by solving (2.7) to certain order, and then substitute this on the right hand side of (2.7) to compute $\lambda$ to the next order, the right hand side of (2.7) has been shown to have a finite $\Lambda \rightarrow \infty$ limit. Nevertheless since under a general change of local coordinates different terms on the right hand side of (2.7) could have their effective $\Lambda$ 's changed differently, there is no guarantee that the right hand side of (2.7) will remain unchanged. In other words, although we have argued that $\lambda$ determined by solving (2.7) has a finite $\Lambda \rightarrow \infty$ limit, it could depend on the choice of local coordinates. Similar arguments show that the right hand side of (3.1) could also depend on the choice of local coordinates. Our goal will be to show that the explicit dependence of the right hand side of (3.1) on the choice of local coordinates through the cut-off $\Lambda$ cancels against the implicit dependence of (3.1) on the choice of local coordinates through $\lambda$, so that the final result for $\Gamma_{\lambda}^{(n)}\left(k_{1}, b_{1} ; \cdots k_{n}, b_{n}\right)$ is independent of the choice of local coordinates.

From now on we shall consider infinitesimal changes in local coordinates so that the $\xi_{A}$ and $\xi_{B}$ in (3.7) are infinitesimal. Using (3.7) the change in $\Gamma_{\lambda}^{(n)}\left(k_{1}, b_{1} ; \cdots k_{n}, b_{n}\right)$ due to a change in $\Lambda$ induced by change of local coordinates take the form

$$
\delta_{\Lambda} \Gamma_{\lambda}^{(n)}\left(k_{1}, b_{1} ; \cdots k_{n}, b_{n}\right)=\sum_{A, B} \Gamma_{\lambda, A}^{(n+1)}\left(k_{1}, b_{1} ; \cdots k_{n}, b_{n} ; 0, \phi\right)\left(\xi_{A}+\xi_{B}\right) \Gamma_{\lambda, B}^{(1)}(0, \phi),
$$

where sum over $A$ and $B$ denotes sum over all Riemann surfaces (including integration over moduli and sum over genus) that contributes to $\Gamma_{\lambda}^{(n+1)}\left(k_{1}, b_{1} ; \cdots k_{n}, b_{n} ; 0, \phi\right)$ and $\Gamma_{\lambda}^{(1)}(0, \phi)$ 
respectively. This can be expressed as

$$
\begin{aligned}
& \left\{\sum_{A} \xi_{A} \Gamma_{\lambda, A}^{(n+1)}\left(k_{1}, b_{1} ; \cdots k_{n}, b_{n} ; 0, \phi\right)\right\} \sum_{B} \Gamma_{\lambda, B}^{(1)}(0, \phi) \\
& +\left\{\sum_{A} \Gamma_{\lambda, A}^{(n+1)}\left(k_{1}, b_{1} ; \cdots k_{n}, b_{n} ; 0, \phi\right)\right\} \sum_{B} \xi_{B} \Gamma_{\lambda, B}^{(1)}(0, \phi) \\
& =\left\{\sum_{A} \xi_{A} \Gamma_{\lambda, A}^{(n+1)}\left(k_{1}, b_{1} ; \cdots k_{n}, b_{n} ; 0, \phi\right)\right\} \Gamma_{\lambda}^{(1)}(0, \phi) \\
& +\Gamma_{\lambda}^{(n+1)}\left(k_{1}, b_{1} ; \cdots k_{n}, b_{n} ; 0, \phi\right) \sum_{B} \xi_{B} \Gamma_{\lambda, B}^{(1)}(0, \phi) .
\end{aligned}
$$

The first term on the right hand side vanishes since $\lambda$ has been chosen so that $\Gamma_{\lambda}^{(1)}(0, \phi)$ vanishes. This allows us to write (3.9) as

$$
\delta_{\Lambda} \Gamma_{\lambda}^{(n)}\left(k_{1}, b_{1} ; \cdots k_{n}, b_{n}\right)=\Gamma_{\lambda}^{(n+1)}\left(k_{1}, b_{1} ; \cdots k_{n}, b_{n} ; 0, \phi\right) \sum_{B} \xi_{B} \Gamma_{\lambda, B}^{(1)}(0, \phi) .
$$

Now suppose $\delta \lambda$ denotes the compensating change in $\lambda$ required to make $\Gamma_{\lambda}^{(1)}(0, \phi)$ vanish with the new choice of local coordinates. Using (2.3) we see that this induces a change in $\Gamma_{\lambda}^{(n)}\left(k_{1}, b_{1} ; \cdots k_{n}, b_{n}\right)$ of the form

$$
\delta_{\lambda} \Gamma_{\lambda}^{(n)}\left(k_{1}, b_{1} ; \cdots k_{n}, b_{n}\right)=\delta \lambda \Gamma_{\lambda}^{(n+1)}\left(k_{1}, b_{1} ; \cdots k_{n}, b_{n} ; 0, \phi\right) .
$$

Adding (3.10) to (3.11) we get the total change in $\Gamma_{\lambda}^{(n)}\left(k_{1}, b_{1} ; \cdots k_{n}, b_{n}\right)$ :

$$
\delta \Gamma_{\lambda}^{(n)}\left(k_{1}, b_{1} ; \cdots k_{n}, b_{n}\right)=\Gamma_{\lambda}^{(n+1)}\left(k_{1}, b_{1} ; \cdots k_{n}, b_{n} ; 0, \phi\right)\left\{\delta \lambda+\sum_{B} \xi_{B} \Gamma_{\lambda, B}^{(1)}(0, \phi)\right\} .
$$

Now setting $n=1$ in (3.12) we get

$$
\delta \Gamma_{\lambda}^{(1)}(0, \phi)=\Gamma_{\lambda}^{(2)}(0, \phi ; 0, \phi)\left\{\delta \lambda+\sum_{B} \xi_{B} \Gamma_{\lambda, B}^{(1)}(0, \phi)\right\} .
$$

$\delta \lambda$ has to be adjusted so that $\delta \Gamma_{\lambda}^{(1)}(0, \phi)$ vanishes since $\Gamma_{\lambda}^{(1)}(0, \phi)$ vanishes both before and after the change. This means that we must have

$$
\delta \lambda+\sum_{B} \xi_{B} \Gamma_{\lambda, B}^{(1)}(0, \phi)=0 .
$$

This in turn makes the right hand side of (3.12) vanish, showing that $\Gamma_{\lambda}^{(n)}\left(k_{1}, b_{1} ; \cdots k_{n}, b_{n}\right)$ remains invariant under a change in the local coordinate system.

Before concluding this section we would like to emphasize one important ingredient underlying our analysis. In arriving at (3.8), (3.9) it was crucial that the change in $\Lambda$ under a change of local coordinates had the form $\xi_{A}+\xi_{B}$ and not a quantity that has general dependence on both $A$ and $B$. This follows from the fact that we used a set of gluing 
compatible local coordinates to define 1PI and 1PR amplitudes. If instead we had chosen a more general local coordinate system - e.g. one where the choice of local coordinate on the puncture of the first Riemann surface depends on the genus of the Riemann surface to which it is being glued - and used it for introducing the cut-off $\Lambda$ then this property will not be respected.

\section{Extension to superstring and heterotic string theories}

We shall now briefly discuss the extension of our analysis to superstring and heterotic string theories when the field $\phi$ arises from the Neveu-Schwarz (NS) sector of heterotic string theory or NSNS sector of type II string theory. Let us for definiteness focus on the heterotic string theory - the generalization to the case of superstrings is straightforward. The analysis proceeds more or less as in the case of bosonic string theory; however in this case the local coordinate system at a puncture requires specifying the holomorphic supercoordinates $(w, \zeta)$ and anti-holomorphic coordinate $\widetilde{w}$. The generalization of (3.2) takes the form [7]

$$
w_{1} w_{2}=e^{-s+i \theta}, \quad w_{2} \zeta_{1}=\varepsilon e^{-(s-i \theta) / 2} \zeta_{2}, \quad w_{1} \zeta_{2}=-\varepsilon e^{-(s-i \theta) / 2} \zeta_{1}, \quad \zeta_{1} \zeta_{2}=0, \quad \widetilde{w}_{1} \widetilde{w}_{2}=e^{-s-i \theta},
$$

with the $s$ integral running from 0 to $\Lambda$ in the definition of the propagator. $\varepsilon$ takes value \pm 1 and we have to sum over both values at the end to implement GSO projection. Now under a change of local (superconformal) coordinates $\Lambda$ still gets shifted by a term of the form $\xi_{A}+\xi_{B}$ as in (3.6), but with $\xi_{A}$ possibly containing even nilpotent parts that depend on the super-moduli of the punctured Riemann surface $A$ and $\xi_{B}$ containing even nilpotent parts that depend on the super-moduli of the punctured Riemann surface $B$. As a result our analysis still goes through, with the sums over $A$ and $B$ in various equations in section 3 now being interpreted to include integration over supermoduli space.

\section{Spurious infrared divergences}

Observations made below (3.1) show that the $n$-point truncated Green's function $\Gamma_{\lambda}^{(n)}$ is equal to $\bar{\Gamma}_{\lambda}^{(n)}$. Since the latter is manifestly free from tadpole divergences, the $n$-point amplitude computed in the shifted background is free from tadpole divergences. However the individual contributions to $\bar{\Gamma}_{\lambda}^{(n)}$ can still suffer from spurious infrared divergence from loop momentum integral since (2.3) (with $\Gamma$ replaced by $\bar{\Gamma}$ on both sides) involves computing amplitudes with zero momentum external $\phi$ legs in the original vacuum. To understand the origin of these divergences and their resolution it will be useful to work with a finer triangulation of the moduli space than the one discussed in [34,35] for dividing the contributions into $1 \mathrm{PI}$ and $1 \mathrm{PR}$ parts. This is done as follows:

1. We begin with a three punctured sphere with some specific choice of local coordinates at the three punctures. The local coordinates are chosen to be symmetric under the exchange of any two of the punctures. 


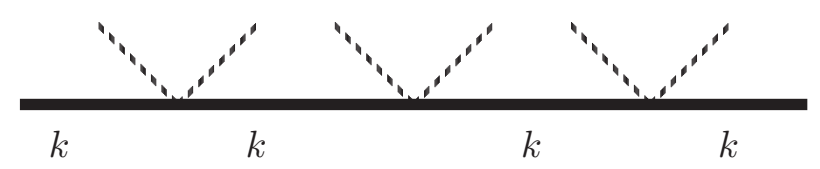

Figure 3. A subdiagram containing spurious infrared divergence. The thick line denotes a massless $\phi$ propagator carrying momentum $k$ and the dashed lines denote $\lambda$ insertions.

2. Now we can generate a family of one punctured tori by gluing two of the punctures of the sphere by the propagator (2.9). We declare these one punctured tori to be composite one punctured tori. The rest of the one punctured tori are labelled as elementary. For the latter we can choose the local coordinate at the puncture in an arbitrary fashion but it must match smoothly to those on the composite one punctured tori across the codimension one subspace of the moduli space that divides composite one-punctured tori from elementary one punctured tori.

3. Similarly by gluing two three punctured spheres across one each of the punctures we can get a family of four punctured spheres. We declare them to be composite four punctured spheres. The rest of the four punctured spheres are declared as elementary. The choice of local coordinates at the punctures of the latter is arbitrary subject to the requirement of symmetry and smoothness across the codimension one subspace of the moduli space that separates the elementary four punctured spheres from composite four punctured spheres.

4. We now repeat the process iteratively. At the end we declare as composite all punctured Riemann surfaces which are obtained by gluing two or more punctures of one or more elementary Riemann surfaces of lower genus and/or lower number of punctures by propagators. The rest of the Riemann surfaces are declared as elementary. The full set of punctured Riemann surfaces contributing to an amplitude are then built in the same way that Feynman diagrams are built by gluing vertices with propagators, with the elementary punctured Riemann surfaces playing the role of the vertices. Indeed this is exactly the way the off-shell amplitudes are constructed in string field theory in the Siegel gauge [23], although we do not assume that the triangulation of the moduli space we are using necessarily comes from any underlying gauge invariant string field theory.

With this triangulation of the moduli space it is easy to see the origin of the spurious infrared divergences. Consider for example the case where we have an internal $\phi$ propagator carrying momentum $k$ propagating in the loop. Then by inserting a pair of external zero momentum $\phi$ through the $\Gamma^{(4,0)}(k, \phi ;-k, \phi ; 0, \phi ; 0, \phi)$ vertex we can increase the number of internal $\phi$ propagators carrying the same momentum $k$ by 1 . Repeating this process we can get a factor of (see figure 3)

$$
\left(1 / k^{2}\right)^{n}
$$

with arbitrary $n$ inside a diagram. For sufficiently large $n$ the integration over loop momentum $k$ will give an infrared divergent contribution, invalidating perturbation theory. 
If we use the language of quantum field theory with all the heavy fields integrated out, then the solution to this problem is clear. Let $c$ denote the net contribution to 1PI 2-point function of the originally masless fields in the shifted background. Then the full propagator is given by

$$
\frac{1}{k^{2}}+\frac{1}{k^{2}} c \frac{1}{k^{2}}+\frac{1}{k^{2}} c \frac{1}{k^{2}} c \frac{1}{k^{2}}+\cdots=\frac{1}{k^{2}-c} .
$$

Thus we can replace the massless propagator by (5.2) and drop all diagrams containing one or more insertions of 1PI 2-point function of massless fields. In this case there are no infrared divergences arising from internal factors of the kind given in (5.1).

The procedure described above requires computing the full 1PI two point function $c$ to a given order for determining the modified propagator, but this is not necessary. Suppose $c$ has a power series expansion in the coupling constant $g$ of the form $\sum_{n \geq 1} c_{n} g^{2 n} \equiv$ $c_{1} g^{2}+\delta c$. Then it is sufficient to use the leading order correction $c_{1} g^{2}$ to define the modified propagator $1 /\left(k^{2}-c_{1} g^{2}\right)$. The full propagator will now have an expansion of the form

$$
\frac{1}{k^{2}-c_{1} g^{2}-\delta c}=\frac{1}{k^{2}-c_{1} g^{2}}+\frac{1}{k^{2}-c_{1} g^{2}} \delta c \frac{1}{k^{2}-c_{1} g^{2}}+\frac{1}{k^{2}-c_{1} g^{2}} \delta c \frac{1}{k^{2}-c_{1} g^{2}} \delta c \frac{1}{k^{2}-c_{1} g^{2}}+\cdots \text {. }
$$

For $k^{2} \sim g^{2}$ the factors of $1 /\left(k^{2}-c_{1} g^{2}\right)$ can become of order $1 / g^{2}$ but each such factor will be accompanied by $\delta c \sim g^{4}$ and hence the successive terms in this expansion will give smaller contributions. ${ }^{8}$ Thus these terms can be treated perturbatively. In fact we can add an arbitrary order $g^{4}$ and higher contribution to $c_{1} g^{2}$ in the definition of the modified propagator and subtract the corresponding contribution from $\delta c$ without changing the final result.

In order to implement this procedure systematically in string theory we proceed as follows:

1. From the triangulation of the moduli space described above it is clear that possible infrared divergent contributions come only from the composite Riemann surfaces where the integration variable $s$ of (2.9) associated with one or more propagators becomes large. To isolate this divergence we split the propagator into its contribution $\Delta_{\text {massless }}$ from massless states ${ }^{9}$ and the rest of the contribution $\widetilde{\Delta}$ :

$$
\Delta=\Delta_{\text {massless }}+\widetilde{\Delta} \text {. }
$$

Note that unlike in the case of (2.11), no cut-off $\Lambda$ on $s$-integration is necesasry for definining $\Delta_{\text {massless }}$ since we are working at non-zero momentum.

2. We also define $\widetilde{\Gamma}$ as the net contribution to an amplitude where all the $\Delta$ 's are replaced by $\widetilde{\Delta}$. $\widetilde{\Gamma}$ defined this way is manifestly free from infrared divergences. Furthermore the full amplitude can now be obtained by gluing $\widetilde{\Gamma}$ 's by the propagator $\Delta_{\text {massless }}$. These are generically infrared divergent from the loop momentum integrals. Note

\footnotetext{
${ }^{8}$ The integration contour of $k$ can always be deformed in the complex plane to make $\left|k^{2}-c_{1} g^{2}\right|$ remain of order $g^{2}$ or larger. Note however that the $k$-integration will introduce non-analytic dependence on the shifted mass which in the present context will translate to non-analytic dependence on the string coupling constant.

${ }^{9}$ Here massless states refer to fields which were massless in the original vacuum. For simplicity we shall ignore the possibility of mixing between physical massless states with unphysical states of the kind discussed in [35], but it should be possible to relax this assumption.
} 
that diagrams where $\Delta_{\text {massless }}$ connects a tadpole to the rest of the diagram vanish by our previous construction since all massless tadpoles have been made to cancel.

3. Let $C(k)$ denote the contribution to the two point function of massless fields carrying momentum $k$ which are 1PI in massless states, i.e. do not contain an internal $\Delta_{\text {massless }}$ propagator that is not part of a loop. If there are more that one massless states then $C(k)$ is a matrix. We now sum over all insertions of $C(k)$ into a propagator by using

$$
\begin{aligned}
& \Delta_{\text {massless }}+\Delta_{\text {massless }} C(k) \Delta_{\text {massless }}+\Delta_{\text {massless }} C(k) \Delta_{\text {massless }} C(k) \Delta_{\text {massless }}+\cdots \\
& \quad=\left(\Delta_{\text {massless }}^{-1}-C(k)\right)^{-1} .
\end{aligned}
$$

The rule for computing the amplitudes to a given order in perturbation theory is now to replace all internal $\Delta_{\text {massless }}$ factors by $\left(\Delta_{\text {massless }}^{-1}-C(k)\right)^{-1}$ with $C(k)$ computed to that particular order, and at the same time drop all contributions to the amplitude that contain a $C(k)$ factor on an internal leg. This renders the amplitudes free from infrared divergence.

4. Note that the computation of $C(k)$ itself could suffer from infrared divergences of the kind discussed above from subdiagrams. Thus the construction of $C(k)$ needs to be carried out iteratively. We begin with the lowest order contribution to $C(k)$ which is free from infrared divergence and define the lowest order modified propagator via (5.5). This is then used to compute the next order contribution to $C(k)$ following the procedure described above. This process is then repeated. In fact since the computation of $\lambda$ via (2.7) also suffers from such spurious infrared divergences, this iterative procedure must be carried out simultaneously for determining $\lambda$ and $C(k)$ to successively higher order.

5. The expression for $C(k)$ obtained this way would in general depend on the choice of local coordinates - only the locations of the poles of the propagator in the $k^{2}$ plane are free from ambiguity [34, 35]. This ambiguity essentially reflects the freedom of moving some contributions from the propagators to the vertices and vice versa. However due to the argument given below (5.3) we expect that the final result for any physical amplitude should not suffer from any ambiguity as long as the procedure we are using renders them free from any spurious infrared divergences.

\section{Acknowledgments}

We thank Rajesh Gopakumar, Michael Green, Boris Pioline, Edward Witten and Barton Zwiebach for useful discussions. The work of R.P. and A.S. was supported in part by the DAE project 12-R\&D-HRI-5.02-0303. A.R. was supported by the Ramanujan studentship of Trinity College, Cambridge, and his research leading to these results has also received funding from the European Research Council under the European Community's Seventh Framework Programme (FP7/2007-2013) / ERC grant agreement no. [247252]. The work of A.S. was also supported in part by the J. C. Bose fellowship of the Department of Science and Technology, India. 


\section{A Effect of shifting a massless field}

Let us consider a quantum field theory containing a massless field $\phi$ and a set of other massless and massive fields. Let us suppose that we have computed the 1PI amplitude involving these fields. Then the truncated Green's functions can be computed by summing up the contributions from tree level graphs with the propagators and vertices constructed from the 1PI amplitudes. We shall study how these truncated Green's functions change when we shift the background value of the field $\phi$ by a constant $\lambda$, without necessarily assuming that the background is a solution to the equations of motion. Our goal will be to prove $(2.3)$.

Now since eventually we shall be interested in setting the background value of $\phi$ to a solution to its equations of motion, or equivalently demand that the tadpole of the field $\phi$ vanishes, it will be natural to demand that all other fields also satisfy their equations of motion. Does this require shifting other fields as well? As in the text, we shall assume that none of the other massless fields need to be shifted even when $\phi$ is shifted, so we only have to worry about a possible shift of the massive fields. To this end we note that only zero momentum modes of the massive fields may be shifted. Since our goal will be to analyze amplitudes where all the external states carry generic non-zero momentum, except possibly some zero momentum $\phi$ fields, we shall integrate out all the zero momentum modes of massive fields, i.e. include in the 1PI amplitude also those which are one particle reducible in one or more zero momentum propagator of massive states. Now the only shift will be in the $\phi$ field, and eventually when we require $\phi$ to satisfy its equation of motion all the massive fields will automatically satisfy their equations of motion.

To proceed we note that (2.3) is equivalent to

$$
\frac{\partial \Gamma_{\lambda}^{(n)}\left(k_{1}, b_{1} ; \cdots k_{n}, b_{n}\right)}{\partial \lambda}=\Gamma_{\lambda}^{(n+1)}\left(k_{1}, b_{1} ; \cdots k_{n}, b_{n} ; 0, \phi\right) .
$$

Thus it is enough to establish (A.1) for arbitrary $\lambda$. To compute the left hand side we shall divide the 1PI action into its kinetic term and the interaction term, and include in the kinetic term only genuine tree level contributions at $\lambda=0$, including the rest of the contribution into the interaction term. Then neither the kinetic terms, nor the $\prod_{i}\left(k_{i}^{2}+m_{b_{i}}^{2}\right)$ terms appearing in (2.1), have any dependence on $\lambda$. Let $\widetilde{\psi}_{b}(k)$ denote the general set of fields in the momentum space. If $V_{\lambda}$ denotes the interaction term in the 1PI action in the shifted background, then, by noting that $\phi$ and $\lambda$ must appear in the combination $\phi+\lambda$ in the interaction part of the 1PI action, we see that

$$
\begin{aligned}
\frac{\partial \Gamma_{\lambda}^{(n)}\left(k_{1}, b_{1} ; \cdots k_{n}, b_{n}\right)}{\partial \lambda} & =\prod_{i=1}^{n}\left(k_{i}^{2}+m_{b_{i}}^{2}\right)\left\langle\prod_{i=1}^{n} \widetilde{\psi}_{b_{i}}\left(k_{i}\right)\left(-\frac{\partial V_{\lambda}}{\partial \lambda}\right)\right\rangle \\
& =\prod_{i=1}^{n}\left(k_{i}^{2}+m_{b_{i}}^{2}\right)\left\langle\prod_{i=1}^{n} \widetilde{\psi}_{b_{i}}\left(k_{i}\right)\left(-\frac{\delta V_{\lambda}}{\delta \widetilde{\phi}(0)}\right)\right\rangle,
\end{aligned}
$$

where \langle\rangle denotes the tree level amplitude computed with the 1PI action. Let us now examine the right hand side of (A.1). This has to be interpreted as the $k \rightarrow 0$ limit of the 
expression where $0, \phi$ is replaced by $k, \phi$. Since the genuine tree level mass of $\phi$ is zero, we have

$$
\Gamma_{\lambda}^{(n+1)}\left(k_{1}, b_{1} ; \cdots k_{n}, b_{n} ; k, \phi\right)=\prod_{i=1}^{n}\left(k_{i}^{2}+m_{b_{i}}^{2}\right)\left\langle\prod_{i=1}^{n} \widetilde{\psi}_{b_{i}}\left(k_{i}\right)\left(k^{2} \widetilde{\phi}(k)\right)\right\rangle .
$$

Now the equation of motion of $\phi$ computed from the 1PI action is

$$
k^{2} \widetilde{\phi}(k)+\left(\frac{\delta V_{\lambda}}{\delta \widetilde{\phi}(-k)}\right)=0 .
$$

Since equation of motion inserted into tree level amplitude computed from 1PI action vanishes for connected Green's function, we get

$$
\prod_{i=1}^{n}\left(k_{i}^{2}+m_{b_{i}}^{2}\right)\left\langle\prod_{i=1}^{n} \widetilde{\psi}_{b_{i}}\left(k_{i}\right)\left(k^{2} \widetilde{\phi}(k)\right)\right\rangle=\prod_{i=1}^{n}\left(k_{i}^{2}+m_{b_{i}}^{2}\right)\left\langle\prod_{i=1}^{n} \widetilde{\psi}_{b_{i}}\left(k_{i}\right)\left(-\frac{\delta V_{\lambda}}{\delta \widetilde{\phi}(-k)}\right)\right\rangle .
$$

Taking the $k \rightarrow 0$ limit of this expression we establish the equivalence of (A.2) and (A.3). This in turn proves (A.1).

We would like to end with the remark that the individual Feynman diagrams contributing to both sides of (A.1) (and (2.3)) are divergent as they may contain zero momentum internal $\phi$-propagators. Thus at this stage our analysis should be taken as a proof of equality of the combinatorial factors which appear when we express the two sides of (2.3) as a sum of Feynman diagrams in the $\lambda=0$ theory. Once the infrared divergences on both sides of (2.3) are regularized using (2.10), it becomes a true algebraic equality.

Open Access. This article is distributed under the terms of the Creative Commons Attribution License (CC-BY 4.0), which permits any use, distribution and reproduction in any medium, provided the original author(s) and source are credited.

\section{References}

[1] M. Dine, N. Seiberg and E. Witten, Fayet-Iliopoulos Terms in String Theory, Nucl. Phys. B 289 (1987) 589 [inSPIRE].

[2] J.J. Atick, L.J. Dixon and A. Sen, String Calculation of Fayet-Iliopoulos d Terms in Arbitrary Supersymmetric Compactifications, Nucl. Phys. B 292 (1987) 109 [INSPIRE].

[3] M. Dine, I. Ichinose and N. Seiberg, F terms and d Terms in String Theory, Nucl. Phys. B 293 (1987) 253 [InSPIRE].

[4] M.B. Green and N. Seiberg, Contact Interactions in Superstring Theory, Nucl. Phys. B 299 (1988) 559 [inSPIRE].

[5] E. Witten, More On Superstring Perturbation Theory, arXiv:1304.2832 [INSPIRE].

[6] N. Berkovits and E. Witten, Supersymmetry Breaking Effects using the Pure Spinor Formalism of the Superstring, JHEP 06 (2014) 127 [arXiv:1404.5346] [INSPIRE].

[7] E. Witten, Superstring Perturbation Theory Revisited, arXiv:1209.5461 [INSPIRE]. 
[8] A. Belopolsky, De Rham cohomology of the supermanifolds and superstring BRST cohomology, Phys. Lett. B 403 (1997) 47 [hep-th/9609220] [INSPIRE].

[9] A. Belopolsky, New geometrical approach to superstrings, hep-th/9703183 [INSPIRE].

[10] A. Belopolsky, Picture changing operators in supergeometry and superstring theory, hep-th/9706033 [INSPIRE].

[11] E. D'Hoker and D.H. Phong, Two loop superstrings. I. Main formulas, Phys. Lett. B 529 (2002) 241 [hep-th/0110247] [INSPIRE].

[12] E. D'Hoker and D.H. Phong, Two loop superstrings. II. The Chiral measure on moduli space, Nucl. Phys. B 636 (2002) 3 [hep-th/0110283] [InSPIRE].

[13] E. D'Hoker and D.H. Phong, Two loop superstrings. III. Slice independence and absence of ambiguities, Nucl. Phys. B 636 (2002) 61 [hep-th/0111016] [INSPIRE].

[14] E. D'Hoker and D.H. Phong, Two loop superstrings. IV. The Cosmological constant and modular forms, Nucl. Phys. B 639 (2002) 129 [hep-th/0111040] [INSPIRE].

[15] E. D'Hoker and D.H. Phong, Two-loop superstrings. V. Gauge slice independence of the N-point function, Nucl. Phys. B 715 (2005) 91 [hep-th/0501196] [INSPIRE].

[16] E. D'Hoker and D.H. Phong, Two-loop superstrings. VI. Non-renormalization theorems and the 4-point function, Nucl. Phys. B 715 (2005) 3 [hep-th/0501197] [INSPIRE].

[17] E. D'Hoker and D.H. Phong, Two-Loop Superstrings. VII. Cohomology of Chiral Amplitudes, Nucl. Phys. B 804 (2008) 421 [arXiv:0711.4314] [INSPIRE].

[18] E. Witten, Notes On Supermanifolds and Integration, arXiv:1209.2199 [INSPIRE].

[19] E. Witten, Notes On Super Riemann Surfaces And Their Moduli, arXiv:1209.2459 [INSPIRE].

[20] E. Witten, Notes On Holomorphic String And Superstring Theory Measures Of Low Genus, arXiv:1306.3621 [INSPIRE].

[21] E. Witten, The Feynman i€ in String Theory, arXiv:1307.5124 [INSPIRE].

[22] R. Donagi and E. Witten, Supermoduli Space Is Not Projected, arXiv:1304.7798 [INSPIRE].

[23] B. Zwiebach, Closed string field theory: Quantum action and the B-V master equation, Nucl. Phys. B 390 (1993) 33 [hep-th/9206084] [INSPIRE].

[24] W. Fischler and L. Susskind, Dilaton Tadpoles, String Condensates and Scale Invariance, Phys. Lett. B 171 (1986) 383 [INSPIRE].

[25] W. Fischler and L. Susskind, Dilaton Tadpoles, String Condensates and Scale Invariance. 2., Phys. Lett. B 173 (1986) 262 [INSPIRE].

[26] B.W. Lee, Renormalization of the $\sigma$-model, Nucl. Phys. B 9 (1969) 649 [INSPIRE].

[27] K. Bardakci, Dual models and spontaneous symmetry breaking, Nucl. Phys. B 68 (1974) 331 [INSPIRE].

[28] K. Bardakci, Dual models and spontaneous symmetry breaking II, Nucl. Phys. B 70 (1974) 397 [InSPIRE].

[29] K. Bardakci and M.B. Halpern, Explicit Spontaneous Breakdown in a Dual Model, Phys. Rev. D 10 (1974) 4230 [INSPIRE]. 
[30] E. Dudas, G. Pradisi, M. Nicolosi and A. Sagnotti, On tadpoles and vacuum redefinitions in string theory, Nucl. Phys. B 708 (2005) 3 [hep-th/0410101] [INSPIRE].

[31] J.J. Atick and A. Sen, Two Loop Dilaton Tadpole Induced by Fayet-Iliopoulos D Terms in Compactified Heterotic String Theories, Nucl. Phys. B 296 (1988) 157 [InSPIRE].

[32] E. D'Hoker and D.H. Phong, Two-loop vacuum energy for Calabi-Yau orbifold models, Nucl. Phys. B 877 (2013) 343 [arXiv:1307.1749] [inSPIRE].

[33] E. D'Hoker, Topics in Two-Loop Superstring Perturbation Theory, arXiv:1403.5494 [INSPIRE].

[34] R. Pius, A. Rudra and A. Sen, Mass Renormalization in String Theory: Special States, JHEP 07 (2014) 058 [arXiv: 1311.1257] [INSPIRE].

[35] R. Pius, A. Rudra and A. Sen, Mass Renormalization in String Theory: General States, JHEP 07 (2014) 062 [arXiv: 1401.7014] [INSPIRE].

[36] J.J. Atick, G.W. Moore and A. Sen, Catoptric Tadpoles, Nucl. Phys. B 307 (1988) 221 [INSPIRE]. 